\title{
Subdiffusion and weak ergodicity breaking in the presence of a reactive boundary
}

\author{
Michael A. Lomholt, ${ }^{1}$ Irwin M. Zaid, ${ }^{2}$ and Ralf Metzler ${ }^{3,1}$ \\ ${ }^{1}$ Physics Department, University of Ottawa, Pavillon MacDonald, Ottawa, Ontario K1N 6N5, Canada \\ ${ }^{2}$ Physics Department, Carleton University, Herzberg Building, Ottawa, Ontario K1S 5B6, Canada \\ ${ }^{3}$ Physics Department, Technical University of Munich, 85748 Garching, Germany
}

\begin{abstract}
We derive the boundary condition for a subdiffusive particle interacting with a reactive boundary with finite reaction rate. Molecular crowding conditions, that are found to cause subdiffusion of larger molecules in biological cells, are shown to effect long-tailed distributions with identical exponent for both the unbinding times from the boundary to the bulk and the rebinding times from the bulk. This causes a weak ergodicity breaking: typically, an individual particle either stays bound or remains in the bulk for very long times. We discuss why this may be beneficial for in vivo gene regulation by DNA-binding proteins, whose typical concentrations are nanomolar.
\end{abstract}

PACS numbers: 05.40.Fb,02.50.Ey,82.20.-w,87.16.-b

The interaction of a diffusive particle with a reactive boundary is of fundamental importance in interface science and technology, e.g., to transport in porous media [1], interactions of proteins with artificial surfaces and membranes 2], or applications such as foam relaxation and surfactants [3]. For a Brownian particle this has been studied extensively, expecially concerning the question how bulk exchange influences the surface distribution of intermittently adsorbed particles [4]. Here, we derive the exchange dynamics with a reactive boundary of a subdiffusing particle, whose unbinding and rebinding times in a molecular crowding environment are both shown to follow long-tailed distributions. We demonstrate a weak ergodicity breaking for the particle trajectory.

This is of particular interest for the search of DNAbinding proteins for their specific binding site on DNA involving successive events of non-specific binding to the DNA and bulk excursions, such that the time spent in either of these events is important in the understanding of the various stochastic mechanisms involved in (bacterial) gene regulation [5, 6, 7]. While the generally applied assumption of Brownian diffusion of proteins works well for typical in vitro experiments under dilute conditions, in vivo the abundance of a multitude of biomacromolecules in the cellular cytoplasm have been shown to cause a state of molecular crowding: large molecules such as proteins, lipids, RNA molecules and ribosomes make up up to $40 \%$ of the cytoplasmic volume [8, 9]. In this superdense environment they hinder each other's motion, causing subdiffusion [10, 11, 12, 13], with a mean squared displacement $\left\langle\mathbf{r}^{2}(t)\right\rangle \propto t^{\alpha} ; 0<\alpha<1$ being a dynamic exponent [14]. By fluorescent methods, subdiffusion was verified for proteins in membranes with $\alpha=0.7$ [10], for proteins in a molecular crowded in vitro environment with $\alpha=0.75$ at higher densities 11], as well as in the cytoskeleton in vivo for messenger RNA of physical size $\approx 100 \mathrm{~nm}$ with $\alpha \approx 0.75[12$ ] and for dextran molecules ranging from $10 \mathrm{kD}$ to $2 \mathrm{MD}$ with $\alpha$ in between 0.59 and 0.84 [13]. The occurrence of subdiffusion for particles with mass as low as $10 \mathrm{kD}$ was also confirmed by computer simulations
13]. The Lac repressor, a typical DNA-binding protein, has $141 \mathrm{kD}[15$, for which a corresponding $\alpha \approx 0.73$ was found [13]. Thus, under molecular crowding conditions, $\alpha \approx 0.75$ seems a fairly standard value for DNA-binding proteins and larger polynucleotides [11, 12, 13]. The time scale over which this subdiffusion persists is not known precisely, but appears to be longer than minutes, so that the following considerations are expected to be relevant for genetic processes [10, 11, 12, 13].

To derive the generalized reactive boundary condition, we pursue a continuous time random walk approach similar to Ref. [16]: A subdiffusing particle jumps from one point to the next after a waiting time distributed according to the long-tailed probability density $\psi(t) \simeq \tau^{\alpha} / t^{1+\alpha}$ $(0<\alpha<1)$ [17]. We start our derivation with the onedimensional lattice, on which $A_{i}$ is the probability to find the particle at lattice point $i=1,2,3, \ldots$ The probability of being at the reactive site (lattice point next to the boundary) is $\mathcal{A}_{0}$, the notation indicating that at site 0 the particle can be exchanged with the bound state with rate $\kappa$. The balance equations then read

$$
\begin{aligned}
& d A_{i}(t) / d t=I_{i}^{+}(t)-I_{i}^{-}(t), \\
& d \mathcal{A}_{0}(t) / d t=I_{0}^{+}(t)-I_{0}^{-}(t)-\kappa \mathcal{A}_{0}(t),
\end{aligned}
$$

and the loss from a given lattice site due to diffusion is

$$
\begin{aligned}
& I_{i}^{-}(t)=\psi(t) A_{i}(0)+\int_{0}^{t} \psi\left(t-t^{\prime}\right) I_{i}^{+}\left(t^{\prime}\right) d t^{\prime} \\
& I_{0}^{-}(t)=\psi_{\kappa}(t) \mathcal{A}_{0}(0)+\int_{0}^{t} \psi_{\kappa}\left(t-t^{\prime}\right) I_{0}^{+}\left(t^{\prime}\right) d t^{\prime},
\end{aligned}
$$

where $\psi_{\kappa}(t) \equiv \psi(t) e^{-\kappa t}$. Substituting for $I^{+}$from Eqs. (10), we rephrase Eqs. (2) in the form $I_{i}^{-}(t)=$ $\int_{0}^{t} \Phi\left(t-t^{\prime}\right) A_{i}\left(t^{\prime}\right) d t^{\prime}$. The kernel $\Phi(t)$ is defined by $\Phi(u)=u \psi(u) /[1-\psi(u)]$ in the Laplace domain, $\Phi(u)=$ $\int_{0}^{\infty} \Phi(t) e^{-u t} d t$ [18]. An analogous relation holds for $I_{0}^{-}(t)$, with the kernel $\Phi_{\kappa}(u)=\Phi(u+\kappa)$. For the gain to site $i$ we have, assuming that the particle jumps to left and right equally likely, $I_{i}^{+}=I_{i-1}^{-} / 2+I_{i+1}^{-} / 2$ and 
$I_{0}^{+}=I_{0}^{-} / 2+I_{1}^{-} / 2$. Note that if the particle attempts to jump left from site 0 , it will be returned back to the same site.

For the continuum limit, we introduce a new timedependent quantity $A_{0}(t)$ by $\int_{0}^{t} \Phi\left(t-t^{\prime}\right) A_{0}\left(t^{\prime}\right) d t^{\prime} \equiv$ $\int_{0}^{t} \Phi_{\kappa}\left(t-t^{\prime}\right) \mathcal{A}_{0}\left(t^{\prime}\right) d t^{\prime}$, corresponding to $\mathcal{A}_{0}(u)=$ $\Phi(u) A_{0}(u) / \Phi_{\kappa}(u)$. Combining above results, we find

$$
\frac{d A_{i}(t)}{d t}=\int_{0}^{t} \Phi\left(t-t^{\prime}\right) \frac{A_{i-1}\left(t^{\prime}\right)+A_{i+1}\left(t^{\prime}\right)-2 A_{i}\left(t^{\prime}\right)}{2} d t^{\prime} .
$$

In the continuum limit $A(x=a i, t)=A_{i}(t) / a$ with the lattice spacing $a$ [19], this equation for $i \geq 1$ yields

$$
\frac{\partial A(x, t)}{\partial t}=\frac{a^{2}}{2} \int_{0}^{t} \Phi\left(t-t^{\prime}\right) \frac{\partial^{2} A\left(x, t^{\prime}\right)}{\partial x^{2}} d t^{\prime}
$$

In the long time limit $u \tau \ll 1, \psi(u) \sim 1-(u \tau)^{\alpha}$, and $\Phi(u) \sim u^{1-\alpha} \tau^{-\alpha}$ to leading order. With $K_{\alpha}=a^{2} /\left[2 \tau^{\alpha}\right]$ and the fractional Riemann-Liouville operator,

$$
{ }_{0} D_{t}^{1-\alpha} A(x, t)=\frac{1}{\Gamma(\alpha)} \frac{\partial}{\partial t} \int_{0}^{t} \frac{A\left(x, t^{\prime}\right)}{\left(t-t^{\prime}\right)^{1-\alpha}} d t^{\prime},
$$

Eq. (4) is equivalent to the fractional diffusion equation $\partial A(x, t) / \partial t=K_{\alpha 0} D_{t}^{1-\alpha} \partial^{2} A(x, t) / \partial x^{2}$ for $x>0[14]$. Similarly, Eq. (1b) can be recast into the form

$$
\begin{gathered}
\frac{d}{d t} \mathcal{A}_{0}(t)+\kappa \int_{0}^{t}\left(\Phi_{\kappa}^{-1} \Phi\right)\left(t-t^{\prime}\right) A_{0}\left(t^{\prime}\right) d t^{\prime} \\
=\int_{0}^{t} \Phi\left(t-t^{\prime}\right) \frac{A_{1}\left(t^{\prime}\right)-A_{0}\left(t^{\prime}\right)}{2} d t^{\prime}
\end{gathered}
$$

In the continuum limit, we recover the expression

$$
\begin{aligned}
-\delta(t) & \mathcal{A}_{0}(0)+\int_{0}^{t} \Psi\left(t-t^{\prime}\right) A\left(0, t^{\prime}\right) d t^{\prime} \\
= & \left.\frac{a^{2}}{2} \int_{0}^{t} \Phi\left(t-t^{\prime}\right) \frac{\partial A\left(x, t^{\prime}\right)}{\partial x}\right|_{x=0} d t^{\prime}
\end{aligned}
$$

with $\Psi(u)=a(u+\kappa) \Phi(u) / \Phi_{\kappa}(u) . \mathcal{A}_{0}(0)$ is 1 , if the particle is initially released at site 0 , and 0 otherwise. The reaction rate at the boundary is $j_{\text {react }}=a \kappa \int_{0}^{t}\left(\Phi_{\kappa}^{-1} \Phi\right)(t-$ $\left.t^{\prime}\right) A\left(0, t^{\prime}\right) d t^{\prime}$, and the right hand side of Eq. (77) represents the flux into $x=0$ from positive $x$. We expand Eq. (7) at $u=0$ in Laplace space (note that $u \ll \kappa[19]$ ]) producing the sought for reactive boundary condition

$$
\left.K_{\alpha 0} D_{t}^{-\alpha} \frac{\partial A(x, t)}{\partial x}\right|_{x=0}=-\mathcal{A}_{0}(0)+k_{0} D_{t}^{-\alpha} A(0, t)
$$

for the subdiffusive particle. This is one of the main results of this work. We defined $k=2 \kappa K_{\alpha} /\left[a \Phi_{\kappa}(u=0)\right] \sim$ $a \kappa^{\alpha}$, using that $\kappa \tau \rightarrow 0$ in the continuum limit [19].

The Berg-von Hippel model maps the binding/unbinding dynamics of a DNA-binding protein to/from the DNA surface onto a cylinder of radius $R_{1}$ placed along the $z$-axis in cylindrical coordinates $(r, \theta, z)$ [5]. For particles subdiffusing in the space $r>R_{1}$ with density $P$ the boundary condition (8) generalizes to

$$
\begin{aligned}
& 2 \pi R_{1} K_{\alpha 0} D_{t}^{-\alpha} \partial P(r, t) /\left.\partial r\right|_{r=R_{1}} \\
& =-P_{0} / L+\left.k_{\text {on } 0} D_{t}^{-\alpha} P\right|_{r=R_{1}}
\end{aligned}
$$

where $k_{\text {on }}=2 \pi R_{1} k$, and we have assumed rotational and translational symmetry around and along the $z$-axis. $L$ is the length of the cylinder along the $z$-axis, and $P_{0}=1$ if at $t=0$ the particle is at the boundary, and $P_{0}=0$ otherwise. The rate of reaction with the cylinder per length along the $z$-axis is $j_{\text {react }}(t)=\left.k_{\text {on } 0} D_{t}^{1-\alpha} P\right|_{r=R_{1}}$, and the fractional diffusion equation (4) is replaced by

$$
\frac{\partial P}{\partial t}=K_{\alpha 0} D_{t}^{1-\alpha} \frac{1}{r} \frac{\partial}{\partial r}\left(r \frac{\partial P}{\partial r}\right)
$$

Consider now the situation when the particle is bound at the boundary at $t=0$. In a crowded environment the full escape of the particle from the boundary consists of two steps: unbinding with rate $\kappa_{\text {off }}$, returning it to the exchange site 0 , and then avoiding to rebind to the boundary such that the particle is unbound at the moment when the environment allows for jumping to site 1 . If the particle is bound at the time when the environment allows a jump (in principle), then the process needs to start over, etc. Thus, the waiting time distribution for the full escape can be written as

$$
\begin{aligned}
& \wp_{\mathrm{unb}}(t)=R(t)+\int_{0}^{t} R\left(t-t^{\prime}\right) \Pi\left(t^{\prime}\right) d t^{\prime} \\
& +\int_{0}^{t} R\left(t-t^{\prime}\right) \int_{0}^{t^{\prime}} \Pi\left(t^{\prime}-t^{\prime \prime}\right) \Pi\left(t^{\prime \prime}\right) d t^{\prime \prime} d t^{\prime}+\ldots
\end{aligned}
$$

with $R(t)=\tilde{\psi}(t)\left[1-\mathrm{P}_{\text {bound }}(t)\right]$ and $\Pi(t)=\tilde{\psi}(t) \mathrm{P}_{\text {bound }}(t)$. Here $\mathrm{P}_{\text {bound }}(t)$ is the probability that the particle is bound at time $t$, given that it was bound at $t=0$ and that the environment has not yet opened up to allow a jump to $i=1$. This yields

$$
\mathrm{P}_{\text {bound }}(t)=\frac{\kappa}{\kappa+\kappa_{\text {off }}}+\frac{\kappa_{\text {off }}}{\kappa+\kappa_{\text {off }}} e^{-\left(\kappa+\kappa_{\text {off }}\right) t} .
$$

The waiting time $\tilde{\psi}(t)$ between opening events in the environment, allowing a jump to $i=1$, is given by

$$
\begin{aligned}
& \tilde{\psi}(t)=\frac{\psi(t)}{2}+\int_{0}^{t} \frac{\psi\left(t-t^{\prime}\right)}{2} \frac{\psi\left(t^{\prime}\right)}{2} d t^{\prime} \\
& +\int_{0}^{t} \frac{\psi\left(t-t^{\prime}\right)}{2} \int_{0}^{t^{\prime}} \frac{\psi\left(t^{\prime}-t^{\prime \prime}\right)}{2} \frac{\psi\left(t^{\prime \prime}\right)}{2} d t^{\prime} d t^{\prime \prime}+\ldots,
\end{aligned}
$$

where the factor $1 / 2$ accounts for the fact that the particle jumps to site 1 only with a probability $1 / 2$ (and stays at 0 with probability $1 / 2$ ). In Laplace space, $\tilde{\psi}(t)$ can be expressed in closed form through a geometric series, 


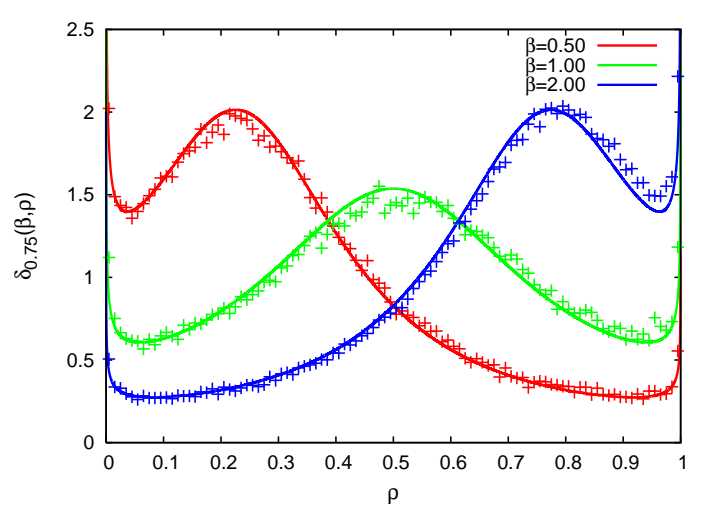

FIG. 1: The distribution $\delta_{\alpha}$, Eq. (18), for various $\beta$, with $\alpha=0.75$. In all cases, a divergence at $\rho=0$ and 1 is observed. The points are results from a stochastic simulation [22].

$\tilde{\psi}(u)=[\psi(u) / 2] /[1-\psi(u) / 2] \sim 1-2(u \tau)^{\alpha}$. Similarly, in the Laplace domain,

$$
\begin{aligned}
& \wp_{\mathrm{unb}}(u)=\tilde{\psi}(u)-\Pi(u)+[\tilde{\psi}(u)-\Pi(u)] \Pi(u) \\
& \quad+[\tilde{\psi}(u)-\Pi(u)] \Pi(u)^{2}+\cdots=\frac{\tilde{\psi}(u)-\Pi(u)}{1-\Pi(u)},
\end{aligned}
$$

where $\Pi(t)$ in Laplace space assumes the exact form

$$
\Pi(u)=\frac{\kappa}{\kappa+\kappa_{\mathrm{off}}} \tilde{\psi}(u)+\frac{\kappa_{\mathrm{off}}}{\kappa+\kappa_{\mathrm{off}}} \tilde{\psi}\left(u+\kappa+\kappa_{\mathrm{off}}\right) .
$$

Collecting the results, we obtain for $\wp_{\text {unb }}$ at small $u$ [19]

$$
\wp_{\mathrm{unb}}(u) \sim 1-\frac{\kappa+\kappa_{\mathrm{off}}}{\kappa_{\mathrm{off}}\left(\kappa+\kappa_{\mathrm{off}}\right)^{\alpha}} u^{\alpha}=1-u^{\alpha} / k_{\mathrm{off}},
$$

where in the continuum limit $\left(\kappa_{\text {off }} \ll \kappa\right)$ we have $k_{\text {off }} \sim$ $\kappa_{\text {off }} / \kappa^{1-\alpha}$. The unbinding times then are distributed according to the power-law $\wp_{\text {unb }} \simeq 1 /\left(k_{\text {off }} t^{1+\alpha}\right)$. This is a central finding of this work: The crowded environment impeding the desorption to the bulk translates the a priori exponential distribution of unbinding times to a power-law 20]. Once arrived at site $i=1$, the particle subdiffuses in the bulk. We consider here the cylindrical case governed by Eq. (10). With initial condition $P_{0}=1$ and a reflecting boundary condition at $r=R_{2}$ [21], an analytic result can be obtained in terms of modified Bessel functions, see Ref. [22] for details. A systematic expansion for small $u$ leads to the result [22]

$$
\wp_{\mathrm{reb}}(u) \sim 1-S u^{\alpha} / k_{\mathrm{on}} .
$$

with the cylindrical cross-section $S=\pi\left(R_{2}^{2}-R_{1}^{2}\right)$. The form $\wp_{\mathrm{reb}}(t) \sim t^{-1-\alpha}$ is typical for subdiffusion [14].

Both unbinding to the volume and returning to the reactive boundary follow power-law forms with identical asymptotic behavior $\sim t^{-1-\alpha}$. The lack of a characteristic time scale separating micro- and macroscopic events gives rise to weak ergodicity breaking [23]. As shown in Ref. 24], the time-averaged probability in the bound state $\bar{p}_{\text {bound }}=\lim _{t \rightarrow \infty} t_{\text {bound }} / t$ for a single trajectory, $\bar{p}_{\text {bound }}$ has the distribution $\mathcal{P}\left(\bar{p}_{\text {bound }}\right)=$ $\delta_{\alpha}\left(k_{\text {on }} /\left(S k_{\text {off }}\right), \bar{p}_{\text {bound }}\right)$, with the Lamperti-generalized $\delta$-function [24, 25]

$$
\delta_{\alpha}(\beta, \rho)=\frac{\pi^{-1} \sin (\pi \alpha) \beta \rho^{\alpha-1}(1-\rho)^{\alpha-1}}{\beta^{2}(1-\rho)^{2 \alpha}+\rho^{2 \alpha}+2 \beta(1-\rho)^{\alpha} \rho^{\alpha} \cos \pi \alpha} .
$$

Note that $\mathcal{P}$ is normalized, $\int_{0}^{1} \mathcal{P}\left(\bar{p}_{\text {bound }}\right) d \bar{p}_{\text {bound }}=1$, and valid in the long $t$ limit. It is independent of $t$ and in that sense an equilibrium is attained. However, while in the Brownian limit $\alpha=1$, ergodicity and a sharply peaked behavior for $\mathcal{P}$ are recovered, the very distinct behavior of $\mathcal{P}$ for $\alpha<1$ is displayed in Fig. 1 for $\alpha=0.75$ : as function of $\rho=\bar{p}_{\text {bound }}$, the distribution peaks at 0 and 1, with a smaller maximum in between. Thus, in a single trajectory a particle is typically either bound or unbound, independently of the duration of the trajectory. This nonergodic behavior is imposed on the system by the probability $\int_{t}^{\infty} \psi\left(t^{\prime}\right) d t^{\prime} \sim t^{-\alpha}$ of never moving, that decays very slowly. The smaller the cross section $S$, the more likely is it to find the particle in a bound state, as it should be. The behavior of $\mathcal{P}$ therefore contrasts the ensemble average over many trajectories, $\left\langle\bar{p}_{\text {bound }}\right\rangle=\left(1+S k_{\text {off }} / k_{\text {on }}\right)^{-1}$, corresponding to the form $\mathcal{P}\left(\bar{p}_{\text {bound }}\right)=\delta\left(\bar{p}_{\text {bound }}-k_{\text {on }} /\left[k_{\text {on }}+S k_{\text {off }}\right]\right)$ [24]. This can be understood as follows. For an ensemble of particles, $k_{\text {on }} / k_{\text {off }}$ defines the nonspecific binding constant $K_{\text {ns }}$, equal to the ratio $N_{\text {bound }} /\left(S N_{\text {unbound }}\right)$ of bound and unbound particles normalized by the cross section [7]. Then $1 /\left(1+S k_{\text {off }} / k_{\text {on }}\right)$ is the ensemble probability that a particle is bound. Weak ergodicity breaking is thus relevant for systems with few particles of a given species.

Transcription factors (TFs), DNA-binding proteins regulating the transcription of a specific gene, occur at very small numbers (a few to some hundred per cell [26]), and in many cases it is essential for the stability of genetic circuits that a TF is always bound at some operator site on the DNA [27, 28]. While the random motion of the TFs in most in vitro experiments is Brownian, molecular crowding in vivo causes subdiffusion of TFs. This would have interesting consequences for gene regulation. Namely, due to the weakly ergodic behavior demonstrated here, TFs will typically stay close to their binding site with a diverging characteristic time scale, such that unbinding and escape to the volume is greatly reduced. The price to pay is that once a TF escapes to the bulk, its return is also affected by an infinite average time. Moreover, there exists a large class of TFs, such as the well-studied Lac and bacteriophage $\lambda$ repressors in E. coli [28], whose specific binding site is located immediately adjacent to their coding region. Biochemical production occurs likely within a few tens of $\mathrm{nm}$ from the coding region 29], and therefore from the targeted binding site. The weak ergodicity breaking thus keeps those TFs within a small volume around their complete 
biochemical cycle, very likely leading to a significant increase in the stability of the regulation of that particular gene. Subdiffusion caused by molecular crowding could therefore be very beneficial for living cells, allowing them to maintain the concentrations of even vital TFs at nanomolar levels. This may significantly impact our current picture of gene regulation in vivo and pose the need to perform experiments much closer to the cellular crowding conditions in order to obtain meaningful information for the in vivo situation.

We derived the generalized reactive boundary condition for the interaction of a subdiffusive particle with a boundary and showed that in the molecular crowding scenario the distribution of unbinding times becomes long-tailed, with the same exponent as the distribution of return times to the boundary. This gives rise to weak ergodicity breaking, relevant for systems with small numbers of diffusing particles. Apart from gene regulation, these effects will impact cellular processes in more general, such as the interactions of biopolymers with membrane proteins, or the exchange of shorter DNA and RNA chains across cellular membranes. Moreover, they will affect trapping phenomena in the vicinity of soft interfaces in more general, e.g., the exchange dynamics from ion clouds in the vicinity of charged or polarized membranes. It should be very interesting to explore these effects by single particle tracking under molecular crowding conditions using fluorescent labelling techniques.

We thank Igor Sokolov and Eli Barkai for helpful discussions, and acknowledge funding by NSERC of Canada and the Canada Research Chairs programme.

[1] S. Stapf, R. Kimmich, R. O. Seitter, Phys. Rev. Lett. 75, 2855 (1995); P. Levitz et al., ibid. 96, 180601 (2006).

[2] L. Vroman, A. L. Adams, G. C. Fischer, and P. C. Munoz, Blood 55, 156 (1980); J. L. Brash et al., ibid. 71, 932 (1988); J. G. Donaldson, R. A. Kahn, J. Lippincottschwartz, and R. D. Klausner, Science 254, 1197 (1991).

[3] A. A. Sonin, A. Bonfillon, and D. Langevin, Phys. Rev. Lett. 71, 2342 (1993); C. Stenvot and D. Langevin, Langmuir 4, 1179 (1988).

[4] See, e.g., O. V. Bychuk and B. O'Shaughnessy, Phys. Rev. Lett. 74, 1795 (1995); R. Valiullin, R. Kimmich, and N. Fatkullin, Phys. Rev. E 56, 4371 (1997).

[5] P.H. von Hippel and O.G. Berg, J. Biol. Chem. 264, 675 (1989), and Refs. therein.
[6] M. Slutsky and L.A. Mirny, Biophys. J. 87, 4021 (2004); M. Coppey, O. Bénichou, R. Voituriez, and M. Moreau, Biophys. J. 87, 1640 (2004); I.M. Sokolov, R. Metzler, K. Pant, and M.C. Williams, Biophys. J. 89, 895 (2005); Y. M. Wang, R. H. Austin, and E. C. Cox, Phys. Rev. Lett 97, 048302 (2006).

[7] M. A. Lomholt, T. Ambjörnsson, and R. Metzler, Phys. Rev. Lett. 95, 260603 (2005).

[8] K. Takahashi, S. N. V. Arjunan, and M. Tomita, FEBS Lett. 579, 1783 (2005); S. Zimmermann and A. Minton, Annu. Rev. Biophys. Biomol. Struct. 22, 27 (1993).

[9] R. J. Ellis and A. P. Minton, Nature 425, 27 (2003); G. Rivas, F. Ferrone, and J. Herzfeld, EMBO Rep. 5, 23 (2004); A. B. Fulton, Cell 30, 345 (1982).

[10] M. Weiss, H. Hashimoto, and T. Nilsson, Biophys. J. 84, 4043 (2003).

[11] D. S. Banks and C. Fradin, Biophys J. 89, 2960 (2005).

[12] I. Golding and E.C. Cox, Phys. Rev. Lett. 96, 098102 (2006).

[13] M. Weiss, M. Elsner, F. Kartberg, and T. Nilsson, Biophys. J. 87, 3518 (2004).

[14] R. Metzler and J. Klafter, Phys. Rep. 339, 1 (2000); J. Phys. A 37, R161 (2004).

[15] A. E. Chakerian and K. S. Matthews, J. Biol. Chem. 266, 22206 (1991).

[16] I. M. Sokolov, M. G. W. Schmidt, and F. Sagués, Phys. Rev. E 73, 031102 (2006).

[17] E. W. Montroll and G. H. Weiss, J. Math. Phys. 6, 167 (1965); H. Scher and E. W. Montroll, Phys. Rev. B 12, 2455 (1975). J. Klafter, A. Blumen, and M. F. Shlesinger, Phys. Rev. A 35, 3081 (1987).

[18] We denote the Laplace transform $f(u)$ of a function $f(t)$ by explicit dependence on the argument.

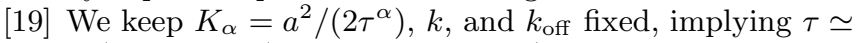
$a^{2 / \alpha}, \kappa \simeq a^{-1 / \alpha}$, and $\kappa_{\text {off }} \simeq a^{1-1 / \alpha}$.

[20] A different scenario corresponding to exponential unbinding in a trapping environment is discussed in Ref. [22].

[21] Crossing of this boundary is viewed to lead the particle into an equivalent cylinder, see O. G. Berg and C. Blomberg, Biophys. Chem. 4, 367 (1976).

[22] I. Zaid, M. A. Lomholt, and R. Metzler (unpublished).

[23] J.-P. Bouchaud, J. Phys. I (Paris) 2, 1705 (1992), G. Bel and E. Barkai, Phys. Rev. Lett. 94, 240602 (2005).

[24] G. Bel and E. Barkai, Phys. Rev. E 73, 016125 (2006).

[25] J. Lamperti, Trans. Am. Math. Soc. 88, 380 (1958).

[26] P. Guptasarma, BioEssays 17, 987 (1995).

[27] See, e.g., A. Bakk and R. Metzler, FEBS Lett. 563, 66 (2004); J. Theor. Biol. 231, 525 (2004), and Refs. therein.

[28] M. Ptashne, A Genetic Switch (Cold Spring Harbor Laboratory Press, Cold Spring Harbor, New York, 2004).

[29] S. Hofmann and O. L. Miller, J. Bacteriol. 132, 718 (1977); P. B. Warren and P. R. ten Wolde, J. Mol. Biol. 342, 1379 (2004). 